\title{
AN EFFECTIVE WAY TO DECREASE PAIN AFTER LOWER EXTREMITY FRACTURE RECONSTRUCTION SURGERIES USING N. ISCHIADICUS BLOCK
}

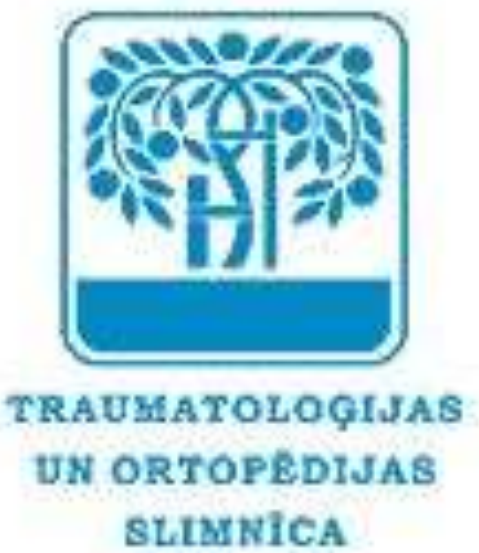
sLIMNicA

\author{
Dr. med. Iveta Golubovska1,2, Arnija Reihmane ${ }^{1}$, Asoc.prof. Aleksejs Miščuks ${ }^{1,2}$ \\ ${ }^{1}$ University of Latvia, Riga, Latvia \\ ${ }^{2}$ Hospital of Traumatology and Orthopaedics, Riga, Latvia
}

Introduction. Spinal anesthesia (SA) is most commonly used performing lower extremity reconstruction surgeries. Peripheral nerve blocks could be method of choice to analgise the region of surgery longer. $\mathrm{N}$. ischiadicus block (NIB) could provide additional analgesia in the perioperative period. The objective of this study is to decrease the post-operative pain level after lower extremity surgeries by using NIB.

Materials and methods. Prospective, randomized study was done in Hospital of Traumatology and Orthopaedics, Latvia. Patients who underwent lower leg fracture reconstruction surgery, were divided in two groups: SA group - SA with Sol. Bupivacaine 2-4ml 0,5\%, depending on BMI; NIB group - SA plus US guided NIB with Sol. Ropivacaine 20-40ml 0,375\%, depending on BMI. Postoperatively all patients received medication by a standardized multimodal analgesia protocol and selfcompleted a pain journal. Rescue morphine was given if the pain $\geq 6$ (NRS). Approval of Ethics committee has been granted. The data was statistically processed using SPSS program.

Results. Overall 48 patients were randomized - 22 in the SA group; 26 in the NIB group. Statistical significance in post-operative pain level were $2 \mathrm{~h}$ after surgery (SA group 1.41 vs NIB group 0.12) and 6h after surgery (SA group 4.68 vs NIB group 2.73) (Image 1). No statistical significance was found regarding to the usage of morphine (Table 1).

Conclusion. The combination of SA and NIB improves the level of analgesia for 6 the first 6 hours after lower extremity surgeries. NIB does not affect the use of morphine.

Table 1

Usage of morphine in SA and NIB group

\begin{tabular}{|c|c|c|}
\hline Patient group & $\begin{array}{c}\text { Mean time untill } \\
\text { the first dose, } \mathrm{h}\end{array}$ & $\begin{array}{c}\text { Mean total dose, } \\
\mathrm{mg}\end{array}$ \\
\hline SA & 6.82 & 24.55 \\
\hline NIB & 7.81 & 25.77 \\
\hline
\end{tabular}

Image 1

Post-operative pain level in SA and NIB groups

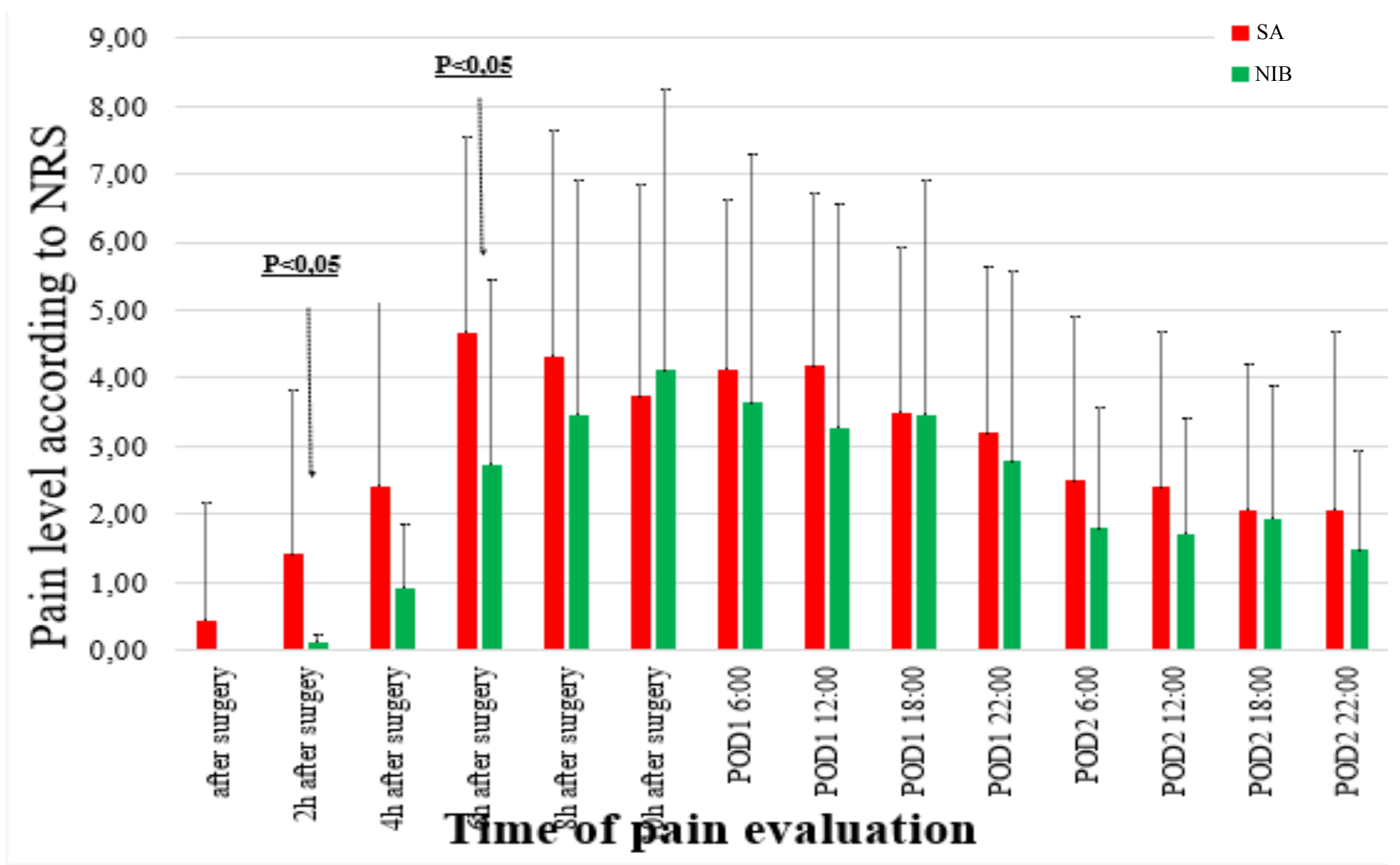

